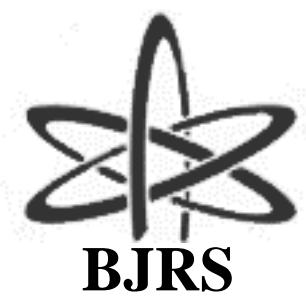

BRAZILIAN JOURNAL

$\mathrm{OF}$

RADIATION SCIENCES

03-1A (2015) 01-11

\title{
Evaluation of a plane-parallel ionization chamber for low- energy radiotherapy beams
}

\author{
A. P. Perini ${ }^{\text {a }}$, L. P. Neves ${ }^{\text {a }}$, W. S. Santos ${ }^{\text {b }}$ L. V. E. Caldas ${ }^{b}$ \\ ${ }^{a}$ Instituto de Física, Universidade Federal de Uberlândia, 38400-902, Uberlândia-MG, Brasil \\ ${ }^{b}$ Gerência de Metrologia das Radiações, Instituto de Pesquisas Energéticas e Nucleares (IPEN-CNEN/SP), \\ 05508-000, São Paulo-SP, Brasil \\ anapaula.perini@ufu.br
}

\begin{abstract}
A plane-parallel ionization chamber, with a sensitive volume of $6.3 \mathrm{~cm}^{3}$, developed at the Calibration Laboratory of IPEN (LCI), was utilized to verify the possibility of its application in low-energy X-ray beam qualities for radiotherapy (T-qualities). This homemade ion chamber was manufactured using polymethyl methacrylate (PMMA) coated with graphite, and co-axial cables. In order to evaluate the performance of this ionization chamber, some characterization tests were performed: short- and medium-term stability, leakage current, saturation, ion collection efficiency, polarity effect and linearity of response. The maximum value obtained in the short-term stability test was $0.2 \%$, in accordance with the limit value of $0.3 \%$ provided by the IEC 60731 standard. The saturation curve was obtained varying the applied voltage from $-400 \mathrm{~V}$ to $+400 \mathrm{~V}$, in steps of $50 \mathrm{~V}$, using the charge collecting time of $20 \mathrm{~s}$. From the saturation curve two other characteristics were analyzed: the polarity effect and the ion collection efficiency, with results within the international recommendations. The leakage current of the ionization chamber was measured in time intervals of 20 minutes, before and after its irradiations, and all the results obtained were in agreement with the IEC 60731 standard. The linearity of response was verified utilizing the T-50(b) radiation quality, and the ionization chamber was exposed to different air kerma rates. The response of the ionization chamber presented a linear behavior. Therefore, all results were considered satisfactory, within international recommendations, indicating that this homemade ionization chamber presents potential routine use in dosimetry of low-energy radiotherapy beams.
\end{abstract}

Keywords:

Ionization Chamber, Low-Energy X-Ray Beam Qualities, Dosimetry 


\section{INTRODUCTION}

Low-energy X-ray beams (10 to $150 \mathrm{kV}$ ) are still utilized in radiotherapy, mainly because of their rapidly attenuation in tissue. This property yields a steep depth dose and makes these Xrays ideal for the treatment of superficial lesions [1]. A study presented by the American Association of Physicists in Medicine (AAPM) indicated a renewed interest in radiotherapy procedures with superficial and orthovoltage X-rays. This conclusion was taken because the number of $\mathrm{X}$ ray machines bought and installed in North America increased in the late 90’s [2].

A large number of centers around the world use low-energy X-rays beams to treat a wide variety of superficial disorders, including superficial malignant lesions [3]. Besides that, low-and medium-energy X-rays beams have been used in intraoperative radiotherapy $[4,5,6]$ or in treatments involving the use of gold nanoparticles, which may lead to a dose enhancement [7]. Although electron beams deliver a more homogeneous dose distribution to large tumors and offer a better preservation of normal tissues, the utilization of low-and medium-energy X-rays in intraoperative radiotherapy procedures has the advantage of placing the radiation unit inside a surgery room because of the relatively modest shielding requirements. Linear accelerators present some advantages in relation to X-ray treatments, mainly because they can target the tumor without affecting the surrounding tissues. However, clinical accelerators are much more expensive than $\mathrm{X}$-ray units, and the low- and medium- energy X-rays still play a role in many developing countries (but mainly in undeveloped countries), where the technology is easier to support and maintain than electron linear accelerators [8].

As low-energy X-rays are still utilized for the treatment of some skin disorders, superficial lesions, and intraoperative radiotherapy, it is important to develop and maintain reference radiation detectors to calibrate dosimeters in these X-ray beams. Ionization chambers are the dosimeters mostly utilized for accurate measurements in radiotherapy beams. They are also recommended for beam dosimetry as reference radiation detectors for calibration procedures $[9,10]$. The ionization chambers have to present some characteristics to be ideal dosimeters; they should present: a homogeneous construction of walls and collecting electrode; no transient electric effects; a collection efficiency higher than 99\%; and they should be indestructible [1, 10]. 
In order to construct an ionization chamber that attend the characteristics of an ideal dosimeter with requirements for a work standard, in this work the development and characterization of a new plane-parallel ionization chamber to be utilized in low-energy X-ray beam qualities for radiotherapy is presented. The main motivation for the development and characterization of this new ionization chamber was the possibility of its utilization at the Calibration Laboratory of IPEN (LCI) as a work standard; and, besides that, the LCI group presents a previous knowledge in the development of ionization chambers for use in diagnostic radiology and radiotherapy energy ranges [11,12].

Furthermore, the ionization chamber designed and developed in this work was planned to be a radiation detector of low cost, easy to assemble, robust, and utilizing only easily commercially available Brazilian materials. The indestructibility characteristic may be included somewhat not seriously, but it must be remembered that an ionization chamber used for calibration purposes has to be robust, with all characteristics well defined. Any change in the active volume of the chamber will result in a change in its calibration factor. To characterize the ionization chamber developed in this work, some tests were undertaken: short- and medium-term stability, leakage current, saturation curve, ion collection efficiency, polarity effect and linearity of response.

\section{MATERIALS AND METHODS}

The plane-parallel ionization chamber manufactured and characterized in this work is presented in Figure 1. The materials utilized in its construction were: polymethylmethacrylate (PMMA) material coated with graphite, silver glue and coaxial cables. The chamber collecting electrode has a diameter of $42.00 \mathrm{~mm}$ and thickness of $2.00 \mathrm{~mm}$, and the chamber walls present a thickness of $2.00 \mathrm{~mm}$. The chamber outer and inner diameters are $56.03 \mathrm{~mm}$ and $46.01 \mathrm{~mm}$, respectively. The interior of the ionization chamber was totally coated with graphite, in order to ensure a homogeneous electric field between the walls and the collecting electrode. 
Figure 1: Photo (a) and (b) diagrammatic scheme of the new plane-parallel ionization chamber developed at LCI where the main components are shown: (A) PMMA wall; (B) Small insulator pieces used to hold the collecting electrode; (C) collecting electrode; (D) stem and (E) sensitive volume.

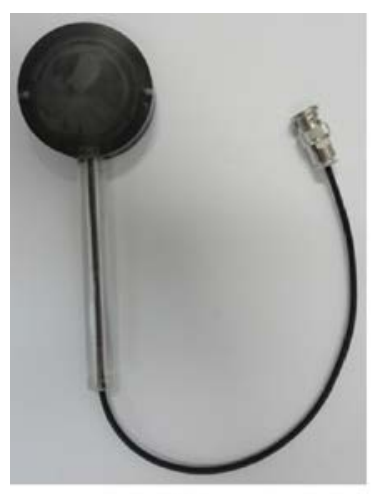

(a)

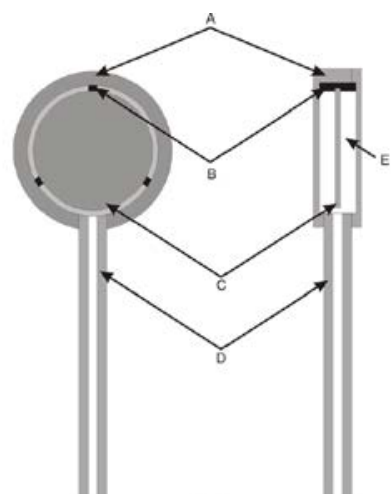

(b)

A ${ }^{90} \mathrm{Sr}+{ }^{90} \mathrm{Y}$ check source device, Physikalisch-Technische Werkstätten (PTW), model 8921, with nominal activity of $33 \mathrm{MBq}$ (1994), was utilized for the stability tests. The other tests were undertaken using an industrial X-ray unit, Pantak Seifert, model ISOVOLT 160HS, that operates from 5 to $160 \mathrm{kV}$.

The established low-energy X-ray beams qualities for radiotherapy, defined by the Bureau International des Poids et Mesures, BIPM [13], were used in this X-ray system, and their parameters are listed in Table 1. The reference system used for these qualities was a parallel-plate ionization chamber, PTW, model M23344. This reference system has traceability to the German primary standard laboratory Physikalisch-Technische Bundesanstalt (PTB).

Tabela 1: Low-energy x-ray beam qualities for radiotherapy, established at the Pantak equipment of LCI for calibration procedures, based on BIPM [13]

\begin{tabular}{|c|c|c|c|c|}
\hline $\begin{array}{c}\text { Radiation } \\
\text { Quality }\end{array}$ & $\begin{array}{c}\text { Voltage } \\
(\mathbf{k V})\end{array}$ & $\begin{array}{c}\text { Additional } \\
\text { Filtration } \\
(\mathrm{mmAl})\end{array}$ & $\begin{array}{c}\text { Half-Value } \\
\text { Layer } \\
\text { (mmAl) }\end{array}$ & $\begin{array}{c}\text { Air Kerma } \\
\text { Rate } \\
\left(\mathrm{mGy}^{\left.-\mathbf{s}^{-1}\right)}\right.\end{array}$ \\
\hline T-10 & 10 & - & 0.043 & $3.130 \pm 0.013$ \\
\hline T-25 & 25 & 0.372 & 0.279 & $2.762 \pm 0.011$ \\
\hline T-30 & 30 & 0.208 & 0.185 & $9.638 \pm 0.042$ \\
\hline T-50(a) & 50 & 3.989 & 2.411 & $0.821 \pm 0.004$ \\
\hline T-50(b) & 50 & 1.008 & 1.079 & $4.027 \pm 0.016$ \\
\hline
\end{tabular}


For the measurements the ionization chamber was attached to an electrometer, model UNIDOS E, PTW, Germany. As the chambers used in this work are unsealed, all measurements were corrected to the reference conditions of temperature $\left(20^{\circ} \mathrm{C}\right)$ and pressure $(101.3 \mathrm{kPa})$.

The uncertainties of all measurements obtained in this work are expanded uncertainties, obtained by the combination of types $\mathrm{A}$ and $\mathrm{B}$ uncertainties, using a coverage factor of 2 .

For the analysis of the results obtained in the present work, the IEC 60731 [14] recommendations, specific for dosimeters utilized in radiotherapy, were used as reference.

\section{RESULTS AND DISCUSSION}

\subsection{Short- and Medium-term Stabilities}

The stability tests were made using a ${ }^{90} \mathrm{Sr}+{ }^{90} \mathrm{Y}$ check source and a support of PMMA specially developed for this ionization chamber (Figure 2(b)) to guarantee the reproducibility in the measurements, as shown in Figure 2. The maximum value obtained in the short-term stability test was $0.2 \%$, in accordance with the limit value of $0.3 \%$ provided by the IEC 60731 standard [14]. The medium-term stability was obtained by plotting the results of the short-term stability test as a function of time. As stated by IEC 60731 [14], the mean value obtained in each short-term test must not differ from the reference value by more than $0.5 \%$. As shown in Figure 3, all deviations were within the acceptable limit [14]. This test was made during a period of three months, and it will be continuously undertaken, within the quality control program of this new radiation detector. 
Figure 2: (a) Experimental set-up and (b) diagrammatic scheme for the stability tests of the new plane-parallel ionization chamber developed at LCI.

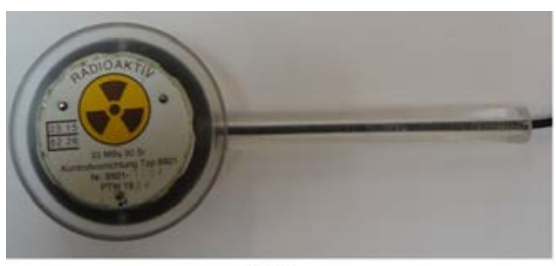

(a)

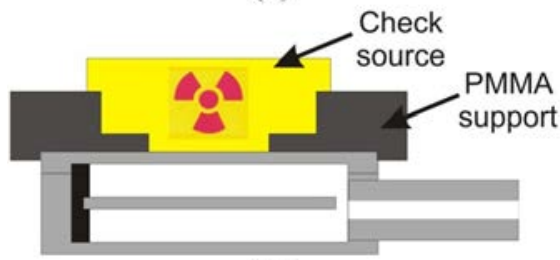

(b)

Figure 3: Medium-term stability test of the new plane-parallel ionization chamber developed at LCI.

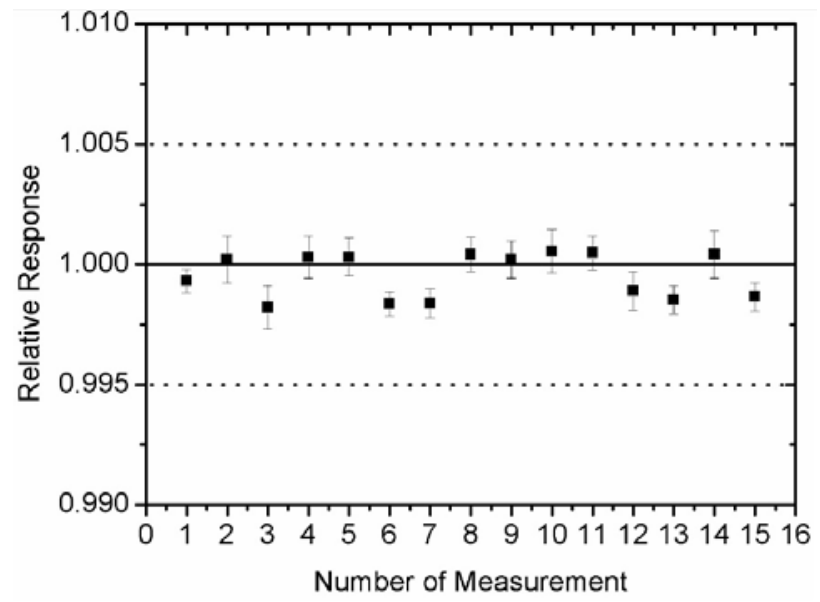

3.2. Saturation, Ion Collection Efficiency and Polarity Effect 
The saturation curve indicates the best tension that shall be applied to the ionization chamber. The saturation curves were obtained utilizing the T-30 and T-50(b) beams for radiotherapy (Figure 4). The value of the collection efficiency for any given irradiation condition is related to the electrode geometry and may be conveniently measured using the two polarity voltages method, given by the following equation [15]:

$$
K_{S}=\frac{\left(\frac{V_{1}}{V_{2}}\right)^{2}-1}{\left(\frac{V_{1}}{V_{2}}\right)^{2}-\left(\frac{M_{1}}{M_{2}}\right)}
$$

Where $\mathrm{M}_{\mathrm{x}}$ is the collected charge at a $\mathrm{V}_{\mathrm{x}}$ voltage, and $\mathrm{V}_{1} / \mathrm{V}_{2}=2$. For $\mathrm{V}_{1}=200 \mathrm{~V}$ (or $-200 \mathrm{~V}$ ) and $\mathrm{V}_{2}=100 \mathrm{~V}$ (or $-100 \mathrm{~V}$ ); the ion collection efficiency was better than $99.99 \%$ for the T-30 and T50(b) radiation qualities.

Figure 4: Saturation curves of the new plane-parallel ionization chamber developed at LCI for $\mathrm{T}-30$ and $\mathrm{T}-50$ (b) radiation qualities. The maximum uncertainty was $0.1 \%$, and therefore not visible in the figure.

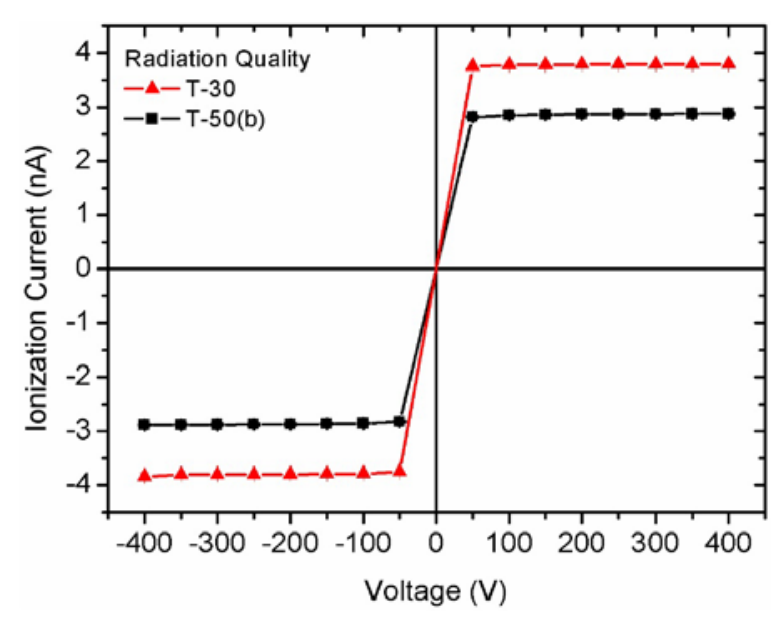

Under identical irradiation conditions, the use of applied voltages of opposite polarities at an ionization chamber may produce different readings; this phenomenon is the polarity effect. It was obtained in this work, by comparing the collected charges at similar voltages of opposite 
signs. The maximum values of the polarity effect obtained for all tested voltages were $0.50 \%$ and $0.25 \%$ for the T-30 and T-50(b) radiation qualities, respectively. These values are within the recommended limit of $1 \%$ [14]. The voltage used in all the tests was $+100 \mathrm{~V}$.

\subsection{Linearity of Response}

The dose response curve of the ionization chamber was obtained using the T-50(b) beam for radiotherapy, with characteristics shown in Table 1. In this test, the tube current was varied from 2 to $25 \mathrm{~mA}$, with the ionization chamber positioned at $50 \mathrm{~cm}$ from the $\mathrm{X}$-ray tube. This variation in the tube current was made in order to provide different air kerma rates that were determined using the reference system calibrated for the T-50(b) quality beam. The results are shown in Figure 5. As expected, the ionization chamber response is linear with the air kerma rate. A linear fit of the chamber response versus air kerma rate was obtained, and the uncertainty in the angular coefficient was only $0.01 \%$, with a correlation coefficient $\mathrm{R}^{2}$ of $0.9999 \%$.

Figure 5: Linearity of response of the new plane-parallel ionization chamber developed at LCI.

Normalization of the chamber response was performed in relation to a tube current of $2 \mathrm{~mA}$.

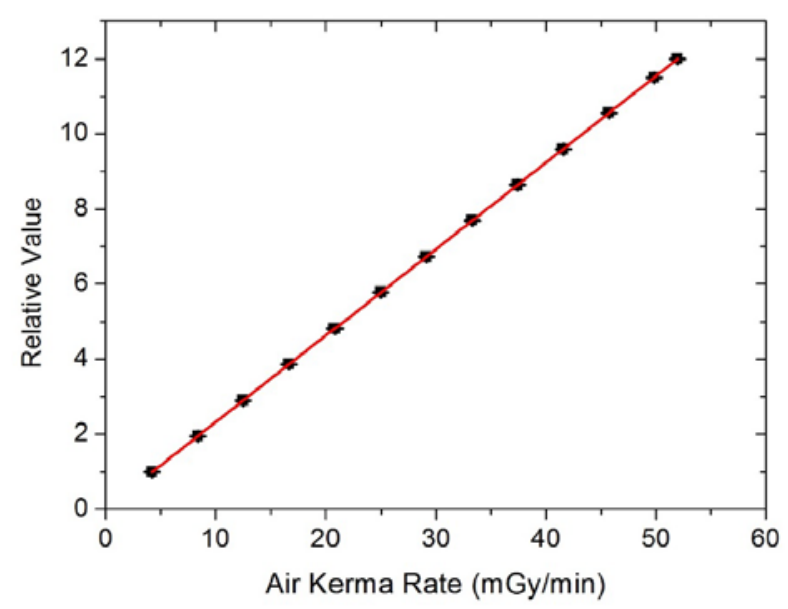

\subsection{Leakage Current}


The leakage current should always be measured and should be insignificant compared to the current obtained during the exposure to radiation. The leakage current was measured before and after the measurements. The maximum value of the leakage current was $0.1 \%$ of the ionization current produced at the minimum air kerma rate produced in this work. This value is within the limit recommended internationally of $0.5 \%$ [14].

\section{CONCLUSIONS}

The parallel-plate ionization chamber designed and developed in this work presented a good performance in all operational tests. The objective proposed in this work was achieved, offering to LCI an alternative work standard for use in low-energy X-ray beams.

The results obtained showed that it is possible to develop a robust ionization chamber in a simple way, using low-cost materials to offer to any metrology laboratory, or user, an accessible way to achieve a dosimeter with requirements for a work standard.

\section{ACKNOWLEDGMENTS}

This work was supported in part by the Brazilian agencies São Paulo Research Foundation (FAPESP, Grants No. 2013/15669-3 and 2013/21741-9) and CNPq, MCT: Project INCT for Radiation Metrology in Medicine.

\section{REFERENCES}

1. HORTON, J. L. Handbook of radiation therapy physics, Englewood Cliffs, N. J: PrenticeHall, 1987.

2. MA, C.M.; COFFEY, C. W.; DEWERD, L. A.; LIU, C.; NATH, R.; SELTZER, S. M.; SEUNTJENS, J. P. AAPM protocol for 40-300 kV X-ray beam dosimetry in radiotherapy and radiobiology. Med Phys, v. 28(6), p. 868-892, 2001. 
3. MALINVERNI, G.; STASI, M.; BAIOTTO, B.; GIORDANA, C.; SCIELZO, G.; GABRIELE, P. Clinical application and dosimetric calibration procedure of the superficial and orthovoltage therapy unit Therapax DXT300. Tumori, v. 88(4), p. 331-337, 2002.

4. DOBELBOWER, R. R.; MITSUYUKI, A. Intraoperative Radiation Therapy, Boca Raton, FL: CRC Press, 1989.

5. KRAUS-TIEFENBACHER, U.; SCHEDA, A.; STEIL, V.; HERMANN, B.; KEHRER, T.; BAUER, L.; MELCHERT, F.; WENZ, F. Intraoperative radiotherapy (IORT) for breast cancer using the Intrabeam system. Tumori, v. 91(4), p. 339- 345, 2005.

6. WENZ, F.; BLANK, E.; WELZEL, G.; HOFMANN, F.; ASTOR, D. ; NEUMAIER, C.; HERSKIND, C.; GERHARDT, A.; SUETTERLIN, M.; KRAUS-TIEFENBACHER, U. Intraoperative radiotherapy during breast-conserving surgery using a miniature Xray generator (Intrabeam): theoretical and experimental background and clinical experience. Women's Health, v. 8(1), p. 39-47, 2012.

7. LEUNG, M. K. K.; CHOW, J. C. L.; CHITHRANI, B. D.; LEE, M. J. G.; OMS, B.; JAFFRAY, D. A. Irradiation of gold nanoparticles by X-rays: Monte Carlo simulation of dose enhancements and the spatial properties of the secondary electrons production Med Phys, v. 38(2), p. 624-631, 2011.

8. MA, C.-M. X-ray therapy equipment, low and medium energy, in Encyclopedia of Medical Devices and Instrumentation, 2nd ed. New Jersey: John Wiley \& Sons, p. 580-590, 2006.

9. KHAN, F. M. The Physics of Radiation Therapy. 1st ed. Baltimore: Willians \& Wilkins, 1984.

10. PODGORSAK, E. B. Radiation Oncology Physics: A Handbook for Teachers and Students. Vienna: International Atomic Energy Agency, 2005.

11. PERINI, A.P.; NEVES, L.P.; VIVOLO, V.; XAVIER, M.; KHOURY, H.J. ; CALDAS, L.V.E. Characterization of a CT ionization chamber for radiation field mapping. Appl Radiat Isot, v. 70, p. 1300-1303, 2012.

12. PERINI, A.P.; NEVES, L.P.; FERNÁNDEZ-VAREA, J. M.; CASSOLA, V.; KRAMER, R.; KHOURY, H.J.; CALDAS, L.V.E. A new parallel-plate graphite ionization chamber as a 60Co gamma radiation reference instrument. Radiat Phys Chem, v. 95, pp. 106-108, 2014. 
13. BIPM - Bureau International des Poides at Mesures. Measuring conditions used for the calibration of ionization chambers at the BIPM. BIPM Rapport BIPM-04/17, Sèvres: BIPM, 2004.

14. IEC - International Electrotechnical Commission. Medical electrical equipment - Dosimeters with ionization chamber as used in radiotherapy. IEC Standard 60731, Genève: IEC, 2011.

15. IAEA - International Atomic Energy Agency. Absorbed dose determination in external beam radiotherapy: an international code of practice for dosimetry based on standards of absorbed dose to water. IAEA TRS 398, Vienna: IAEA, 2001. 